\title{
RECAIDAS EN LA FIEBRE TIFOIDEA TRATADA CON CLOROMICETINA
}

\author{
Prof. JULIO MENEGHELLO R. y Drs. OSCAR UNDURRAGA A. \\ y ALFREDO RAIMANN N. \\ Cátedra de Pediatria Profs. Nrturo Baça Goñi y Julio Meneghello R. \\ "Hospital Mancel Arriarán".
}

La cloromicetina constituye el mejor tratamiento actual de la fiebre tifoidea. Esto ha sido repetidamente probado tanto en publicaciones extranjeras como nacionales " " ", ", ", Sin embargo, ella no ha logrado resolver algunos aspectos importantes de la enfermedad, tales como las complicaciones causantes de la mortalidad, el portador que significa un problema epidemiológico no resuelto y las recaídas, a las que deseamos referirnos en esta comunicación.

Es indispensable previamente definir los términos recaida y recidiva. Por recaída se entiende la reaparición de toda - parte de la sintomatología clínica de la enfermedad después de transcurrido un periodo afebril inferior al período de incubación considerado en su límite máximo y recidiva es la repetición de la sintomatología después de un periodo afebril superior al límite máximo del período de incubación, siendo en realidad una verdadera reinfección.

No se ha obtenido una variación substancial en la frecuencia de las recaídas en la fjebre tifoidea con el uso de la cloromicetina. Las estadisticas anteriores al uso de este antibiótico daban valores que fluctuaban entre 6,5 y $30 \%$. John " publica la frecuencia de recaídas obtenida por diferentes autores, la que alcanza un $26 \%$, eso sí que en este material están incluidos los primeros casos tratados, que fueron los que dieron el mayor porcentaje de recaidas " $"$,". Entre nosotros. Meneghello y Hasbun en una publicación sobre el tema 5 encuentran una frecuencia de $9,5 \%$. Una revisión de 500 casos de fiebre tifoidea en adultos hecha por Kraljevic y colaboradores" arroja un $8,6 \%$ de recaídas.

Nuestro estudio comprende 30 casos de recaída de fiebre tifoidea que corresponden a 28 enfermos, ya que dos de ellos tuvieron dos recaidas. Este grupo se obtuvo de 314 casos de fiebre tifoidea que hemos logrado tratar con cloromicetina desde Mayo de 1949, fecha en que ini- ciamos este tratamiento hasta Abril de 1953. Como puede observarse en la tabla No 1 , el mayor porcentaje de recaídas correspondió al primer año en que usamos este tratamiento y ello se debió, como lo expusimos en publicaciones anteriores "i, a que debido a la escasa disponibilidad de la droga los tratamientos se hacían por plazos insuficientes. Desde que usamos la cloromicetina por plazos más prolongados, hasta 14 dias, vimos disminuír la frecuencia de las recaídas. Ultimamente, desde hace más o menos un año, hemos acortado a sólo 10 días el tratamiento, con el objeto de obtener un mayor rendimiento de la droga y de las camas disponibles para esta enfermedad, no habiendo comprobado con ello un aumento en el número de recaídas. El porcentaje de recaídas que tuvimos alcanzó a $8,9 \%$, valor muy semejante al obtenido en adultos por Kraljevic y colaboradores :

En la tabla No 2 se presentan algunas características generales de nuestros enfermos. La mayoría tenían 7 o más años de edad. El promedio de días, con que los enfermos ingresaron, alcanzó a 12, Ia dosis promedio de cloromicetina usada fué 16,7 grs. y la duración del tratamiento, 13,1 días. E1 momento de aparición de la recaída en relación con la normalización de la temperatura, fué a los 16 días, variando este plazo entre 6 y 31 días.

$$
\text { TSIRI,A }>1
$$

FIERRE TIFOIDEA TRATADA CON CLOROMICETINA Y FRECUENCIA DE RECAIDAS. OBSERVACION

\begin{tabular}{|c|c|c|c|}
\hline & Tifoideas tcatadas & Kecaídas & $\because o$ \\
\hline 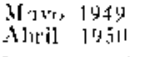 & $1:$ & $i$ & $3.3,5$ \\
\hline 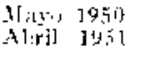 & 16.3 & 7 & 6,6 \\
\hline $\begin{array}{l}\text { Maso } 1451 \\
\text { Aln:j J452 }\end{array}$ & $2 r_{3}$ & 6 & $F, R$ \\
\hline 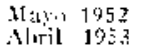 & 114 & 9 & 7.8 \\
\hline TOTAl, & $31 t$ & 29 & 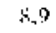 \\
\hline
\end{tabular}




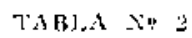

ALGUNAS CARACTERISTICAS DE 28 ENFERMOS IE FIEBRE TIFOIDEA TRATADOS CON CLOROMICETINA Y QUE SUFRIERON RECAIDA

\begin{tabular}{|c|c|c|c|c|c|c|c|c|c|}
\hline \multicolumn{2}{|c|}{$E d a d$} & \multicolumn{2}{|c|}{ Sexo } & \multicolumn{3}{|c|}{$\begin{array}{l}\text { Tipo clínico de la } \\
\text { Fiebre Tifoidea }\end{array}$} & \multirow{2}{*}{$\frac{\begin{array}{c}\text { Dizs enferm. antes } \\
\text { del tratamiento }\end{array}}{\text { (promedio) }}$} & \multirow{2}{*}{$\begin{array}{c}\text { Dosis usada } \\
\text { en grs. } \\
\text { (promedio) }\end{array}$} & \multirow{2}{*}{$\frac{\begin{array}{c}\text { Dutación trat. } \\
\text { en dias }\end{array}}{\text { (promedio) }}$} \\
\hline $\begin{array}{l}6 \text { a. o } \\
\text { menos }\end{array}$ & ]$_{\text {más }}^{a}$ & $s$ & 7 & leve & motiana & grave & & & \\
\hline 3 & 25 & 18 & 10 & 8 & 7 & 13 & 12 & 16,7 & 13,1 \\
\hline
\end{tabular}

La iniciación de la recaida se tradujo por la elevación de la temperatura, la que en la mayoría de los casos, 18 oportunidades, la vimos ascender en forma brusca siendo el ascenso en los 12 restantes en forma insidiosa. En el gráfico $N^{\circ} 1$ se muestran los tipos de curva térmica observados: en 14 enfermos la curva fué irregular, en 10 de tipo remitente, en 3 irregular y remitente y en 3 oportunidades en aguja. La duración de la curva térmica no tuvo diferencia apreciable entre

I Curvas Termicas en Recaidas de Fiebre Tifoidea

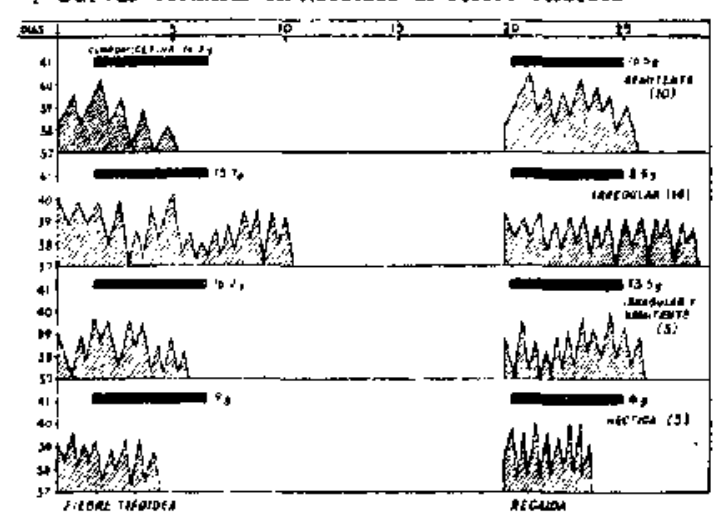

Gráfico $N^{0} 1$

las recaidas que tratamos con cloromicetina y las que no recibieron tratamiento, $\mathbf{8 , 5}$ y $\mathbf{9 , 5}$ días, respectivamente. La caída de la temperatura, a semejanza de lo que ocurrió en la enfermedad de origen, fué en crisis en 8 oportunidades $y$ en lisis en 22 .

En la tabla No 3 se anotan los sintomas clínicos más destacados que pudimos apreciar en nuestros enfermos. El compromiso del estado general varió desde poco aparænte, lo que ocurrió en 18 oportunidades, de mediana intensidad en 5 casos e intenso en 7. En algunas ocasiones la gravedad fué tan manifiesta que alcanzó hasta el estado tífico. La Iengua en la mayor parte de los enfermos volvió a hacerse saburral y en 5 casos no presentó alteraciones. Las roseolas pudieron comprobarse en 3 recaídas, siendo su frecuencia muy inferior a la presencia del sintoma en la enfermedad original. Lo mismo se puede decir en cuanto a los síntomas bronquiales, los que siendo muy frecuentes en la fiebre tifoidea aparecieron en las recaídas sólo en tres oportunidades. La sintomatología cardíaca más frecuentemente encontrada fué la aparición de soplos, de preferencia sistólicos y localizados en diferentes zonas del área cardíaca, en general poco intensos y de carácter fugaz. El apagamiento de los tonos sólo se consignó en dos oportunidades. Hubo una enfermita que en una segunda recaída presentó una miocarditis clínica y electrocardiográfica que la llevó a la insuficiencia cardiaca, de la cual se recuperó. El dolor abdominal no se anotó en ningún enfermo, en cambio el meteorismo estuvo presente en 13 oportunidades, siendo intenso sólo en dos casos. La hepato y esplenomegalia fueron signos pesquisados con bastante frecuencia en las recaidas, observandose en 14 enfermos.

Quisimos conocer la evolución de las reacciones de aglutinación en las recaídas. En 20 oportunidades ellas fueron positivas; de ellas, 10 eran ya positivas en la enfermedad original, 2 que eran negativas se hicieron positivas $y$ finalmente en 7 se observó aumento del título. Re-

TABJ.A XI"

SINTOMAS CLINICOS EN 30 RECAIDAS DE FIEBRE TIFOIDEA

\begin{tabular}{|c|c|c|c|c|c|c|c|c|c|c|c|c|c|}
\hline \multicolumn{3}{|c|}{$\begin{array}{c}\text { Compromigo } \\
\text { Eemeral }\end{array}$} & \multicolumn{2}{|c|}{ Lergua } & \multirow[t]{2}{*}{ Roseola } & \multirow[t]{2}{*}{ Eronquitis } & \multicolumn{2}{|c|}{ Corazón } & \multicolumn{3}{|c|}{ Meteorismo } & \multirow[t]{2}{*}{ Hepatom. } & \multirow[t]{2}{*}{ Fsplenom. } \\
\hline+ & $+t+$ & $\because \div \div$ & Sab. & Norm. & & & Alt. & Norm & + & $\div+\cdots$ & +4 & & \\
\hline 13 & 5 & 7 & 25 & 5 & 3 & 3 & 12 & 18 & 9 & 2 & 2 & 14 & If \\
\hline
\end{tabular}




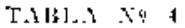

REACCIONES DE AGLUTINACION EN 27 ENFERMOS DE FIEBRE TIFOIDEA TRATADOS CON CLOROMICETINA Y QUE SUFRIERON RECAIDA

\begin{tabular}{|c|c|c|c|c|c|}
\hline $\begin{array}{l}\text { Se mantienen } \\
\text { positivas }\end{array}$ & $\begin{array}{l}\text { Se tuacen } \\
\text { positjyas }\end{array}$ & $\begin{array}{c}\text { Aumentan } \\
\text { título }\end{array}$ & $\begin{array}{c}\text { Dismpinuyen } \\
\text { título }\end{array}$ & $\begin{array}{c}\text { Se mantıetuerl } \\
\text { negativas }\end{array}$ & $\begin{array}{l}\text { Se hacen } \\
\text { negativas }\end{array}$ \\
\hline 10 & 3 & 7 & $l$ & 4 & 3 \\
\hline
\end{tabular}

acciones de aglutinación negativas se consigna en 7 recaidas; de ellas, 4 ya eran negativas y 3 que eran positivas se hicieron negativas (Tabla No 4).

En la tabla $N^{0} 5$ se observa el resultado de los hemo, copro y urocultivos, así como también de los hemogramas. Destaca la relativa frecuencia de los hemocultivos positivos, así como el hemograma en un número apreciable de recaídas, con características semejantes al de la enfermedad original.

Del total de enfermos que recayeron, 20 fueron tratados con cloromicetina usando los díferentes esquemas que han sido dados a conocer en publicaciones anteriores. Tres enfermos fueron tratados según el esquema original de Woodward, 3 con horario cada 12 horas $y$ dosis inicial y la mayoria, 14 con horario cada 12 horas $\mathrm{y}$ sin dosis inicial. La prolongación del tratamiento en promedio fue 10,9 días $y$ la dosis promedio en gramos, 12,1 (tabla Ne 6). La iniciación del tratamiento en relación a la elevación de la temperatura se efectuó en un plazo promedio de 4,7 días $y$ se consiguió la normalización de la temperatura en un promedio de 3,8 días.

En la mayoría de los casos la recaída se presentó como una enfermedad benigna, de corta duración y en la que la sinto- matología clínica alcanzó poca signíficación. Sin embargo, hubo un grupo de 5 enfermos en los que ella cobró particular intensidad $\mathrm{y}$ hasta se presentaron complicaciones. Esta ha sido la razón por la que actualmente sometemos a tratamiento con cloromicetina a toda recaída por muy benigna o fugaz que aparezca, ya que no tenemos ningún medio para prever la evolución que ella va a seguir. Por considerarlo de interés, expondremos en forma somera la observación de los enfermos con recaída y que sufrieron complicación (gráfico $\mathrm{N}^{\circ}$ 2):

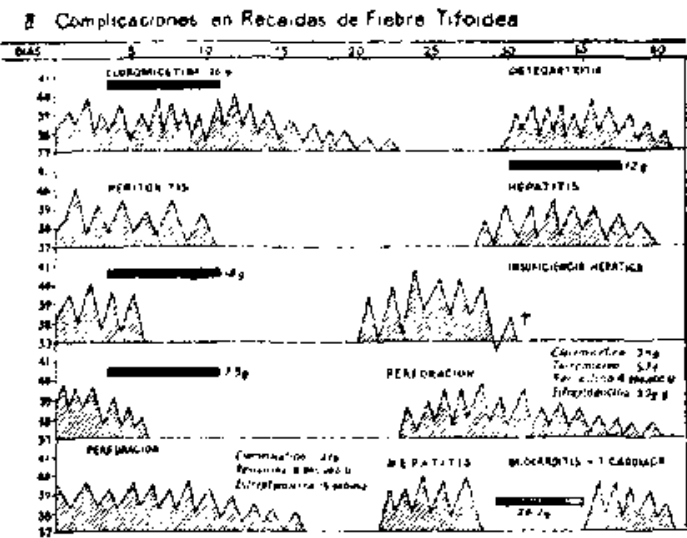

Gráfico $\mathrm{N}^{9} 2$

TABI.A X"

HEMOCULTIVO, COPROCULTIVO, UROCULTIVO Y HEMOGRAMA EN RECAIDAS DE FIEBRE TIFOIDEA

\begin{tabular}{|c|c|c|c|c|c|c|c|c|c|}
\hline \multicolumn{2}{|c|}{ Hemocultiva } & \multicolumn{2}{|c|}{ Coprocultivo } & \multicolumn{2}{|c|}{ Urocultivo } & \multicolumn{4}{|c|}{ Hermograma } \\
\hline$\div$ & $(\rightarrow)$ & + & $(-j)$ & $t$ & $(-)$ & Anemì & Lelicop. & Desv. izq. & Aneosinof \\
\hline 7 & 20 & + & $2 ?$ & 2 & 25 & 16 & 20 & 14 & 2.1 \\
\hline
\end{tabular}

TABHA $\therefore:$,

TRATAMIENTO CON CLOROMICETINA EN RECAIDAS DE FIEBRE TIFOIDEA

\begin{tabular}{|c|c|c|c|c|c|}
\hline \multirow{2}{*}{$\begin{array}{l}\text { Duración en dias } \\
\text { (promedio) }\end{array}$} & \multirow{2}{*}{$\begin{array}{l}\text { Dosis en Ers. } \\
\text { (premedio) }\end{array}$} & \multicolumn{4}{|c|}{ Esquena de tratamiento } \\
\hline & & Woodward & $\begin{aligned} \text { Cada iz horas con } & \text { dosis inicial }\end{aligned}$ & Cada 12 & $\begin{array}{l}\text { horas sio } \\
\text { inicial }\end{array}$ \\
\hline 10,9 & 12,1 & 3 & $i$ & & 4 \\
\hline
\end{tabular}


S. M. A. $: 2$ años. Obs. N: $56537-49$. Enfermo que presenta una fiebre aifoidea grave de l 1 días de crolación. que lrabada con cloromicerina según es. quema original de Woodwatd $\gamma$ con dosis colal de 16 grs. en 8 dias, mejori sn an piazo extracodinariamenic prolongado. alrededor de 3 semanas. En la $5^{\prime \prime}$ semana presenta una esteontritis de la metiena izquierda que se catalogó canwe tifica por encontrarse el bacilo de Jiberth en el liquido de la punzión articular. Esta complicación no recibio leacamicnto con eloromicti. na, y crolucionó fasotablemente un un plazo prolongaclis, de 53 dias.

H. D. C. 7 años. Obs N'69815.50. Enfern:o que cn la segunda semana de cvolución de una fubre tifoidea. presenta una peritonitis generalizada por perforación. la gue es intervenida mejorando en an plazo de 28 dias: no feli 1 rilada con ctoromicetina por no disponerse enconces de droga. Cinco dias sespués de ser dado de alta, presenta fiebre de tipo rénitente. bepatoespletemegalia istericia. con alteración de las pruebas de fleculacion y aumento de ia bilirrubinemia direca. La temperatura elevads persistió duranie nueve dias $y$ descendio en lisis al día siguicute de inicialo el tratamiento con cloromicetina. La iclericia daris docz dias. Jivolucionó en genetal en forma stustactoria

R. N. M. 9 arios. Obs. $\Lambda^{9} 52679$. Presenta una fiehre tifoidea de mediana gravedad, que. tratada con coromicetiona durate un plazo insuficicnte. hase una recaida con hepatitis grave y sinturnas de insuliciencia hepatica. a consecuentia de la cual fallece. La recaía a no fuc trasada con cloromicetina. La autopsia reveló reparación cvidente de las lesiones intestinales $c$ infiltucion grasosa intensa y dilusa del hígado.

J. C. S, 9 años. Obs. N'? $1178+5-53$. Fiebes Lifoida grave que preserta una panbemocitopenia y que responde in forma satisfactcria al tratimiento con cloromicetina cada doce horas. Dispuis de permanecer doce dias af́bril. presenta una recaida grave que se complica de parforación intestinal la que es operida tardiamente. a las 58 horas. hacisindose simultancamente un 1 ratamiento interso con antibioticos. Mejors desputs de una erolución truy prolongada a consecuencias de una fistula intestinal, la que espontaneamente se va reduciendo.

O. A. G. 7 años. Olss b7721.50. Enfirmita que prisents una liebre tifoided grave. que se perfora en la cuarta semana de evolución. se interviene compro. bandose una peritonitis $y$ se traca inlensamenre con penicilina, estreptomicina, cloronicetina \& hidratacion parenteral, evolucionando bicn. A los 20 dias de haber sido dada de alta. apares fiebre c ictericia gratve con sindrome putpúrico y compromiso psiquico. cuadro que se interpreta como una recaida, la cual no fue tralada con cloromicetina $y$ evolucionó saisfacrotiamente en veinle días. đurando el cuadro febtil alrededor de una semana. Ttes semanas mís tarde pre. senta noter alpa termica con coprocultivo positivo para el batilo de Eberth, dominando en esta ocasión Jos signos de gran compromiso miocardica tanlo dinicos como clectrocardiográficos y que jevin a l, enferma a la insuficienzia cardiaca. La efolución fue satisfactoria en un plazo de 1 l dias. En esla segunda recaida recibe tratamienco con cloromicetina on desis y pur plazo que estumamos suficiente,

\section{RESUMEN}

Se analizan en el presente trabajo 30 rccaídas de ficbie tifoidea obtenidas de un material de 314 enfermos en un perio de 4 años (Mayo de 1949 a Abril de 1953 ! y que habian sido tratados con cloromicetina. Se exponen algunas caracteristicas generales de este grupo, se revisa la sintomatología tanto clínica como de laboratorio, se da a conocer su tratamiento y, finalmente, se analizan las complicaciones aparccidas en el curso de las recaídas.

\section{CONCLUSIONES}

1. La recaida de la fiebre tifoidea tratada con cloromicetina es de observación relativamente frecuente, $8,9 \%$ en nuestro material.

2. Ella apareció en un plazo promedio de 16 dias en relación con la normalización de Ia temperatura.

3. Se presentaron las recaídas de preferencia en aquellos casos de fiebre tifoideo que tuvieron evolución grave.

4. La sintomatología clinica fué semejante a la de la fiebre tifoidea pero, en general, más atenuada que la de ella. Destaca la frecuencia con que se apreció la hépato-esplenomegalia.

5. Las reacciones de aglutinación mostraron tendencia a mantenerse positivas - a aumentar su título. En sólo 3 oportu. nidades ellas se hicieron negativas duran. te la recaida.

6. En 7 reeaídas se pesquisó un hemocultivo positivo, en 4 coprocultivo positivo $y$ en 2 urocultivo positivo.

7. El hemograma, en la mayoria de los enfermos, presentó características semejantes al hemograma de la fiebre tifoidea.

8. No se observó un aumento en la frecuencia de las recaidas con la reducción del tratamiento de cloromicetina a 10 días.

9. Del total de 30 recaidas, 5 presentaron complicaciones, algunas graves, y aún, en una oportunidad, mortal. 
10. No se previno la aparición de recaídas con la práctica de la vacunación antitífica que realizamos sistemáticamente en todos nuestros enfermos una vez normalizada la temperatura.

11. El tratamiento se practicó en 20 enfermos con la misma dosis de cloromicetina y por el mismo plazo que en Ia fiebre tifoidea $y$, dada la proporción de recaidas que sufren complicaciones, se aconseja su tratamiento con cloromicetina.

\section{SUMMARY}

1. The relapse of the typhoid fever treated with chloromycetin is of relatively frequent observation, $8.9^{\%} / \mathrm{in}$ our $\mathrm{ma}-$ terial.

2. It appeared in an average term of 16 days in relation with the normalization of the fever.

3 . The relapses presented were preferable in those cases in which typhoid fever had dangerous evolution.

4. The clinic symtomatology was similar to that of the typhoid fever, but in general, more diminished than it. It detaches the frequency with which it was appreciated an increase in the size of the liver and spleen.

5. The reactions of agglutination showed tendency to maintain themselves positive or increase their title. In only 3 occasions during the relapse these were made negative.

6. In 7 relapses a positive culture of the blood was searched, in 4 culture of the feces and in 2 positive culture of the urine.

7. The blood picture in the mayority of the patients presented characteristics similar to that of the typhoid fever.

8. No increase in the frequency of the relapses was observed with the reduction of the treatment of chloromycetin for 10 days.

9. Out of the total of 30 relapses, 5 presented complications, some dangerous and even mortal in one occasion.

10. The appearance of relapses was not forseen with the practice of the antityphoid vaccination which we made systematically in all our patients once. the temperature was normalized.

11. The treatment was practised in 20 patients with the same doses of chloromycetin and for the same length of time as in the typhoid fever and due to the amount of relapses which suffered complications, a treatment with chloromycetin is advisable.

\section{BIBLTOGRAF'́A}

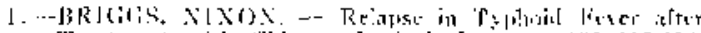

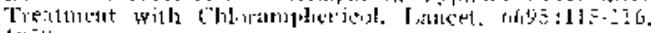
(1)

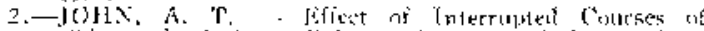

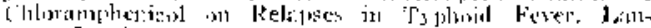
ct, 6?3: $: 257,1952$.

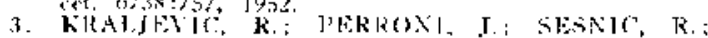

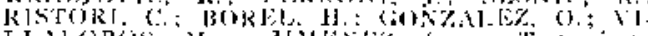

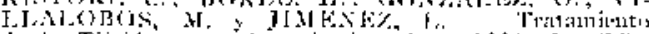

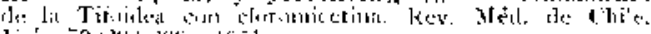
tid. 79 :34.i-390, 1951.

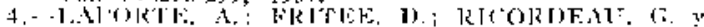

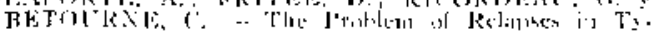

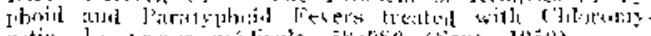

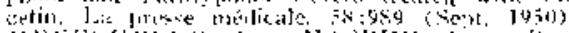

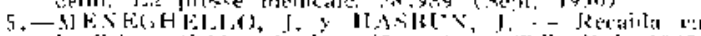

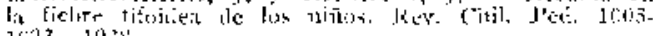
$1123,19,3$

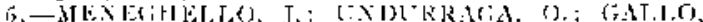

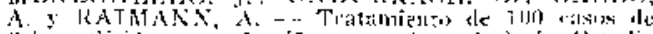

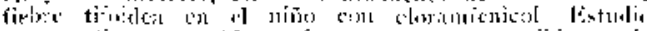

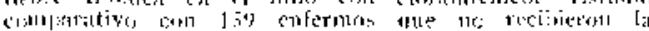

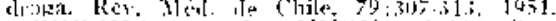

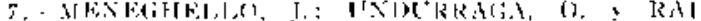

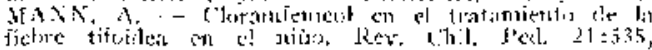
(1) $3(1$.

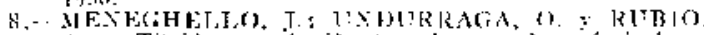

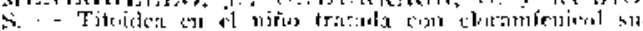

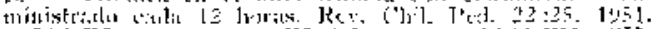

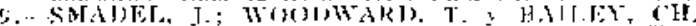

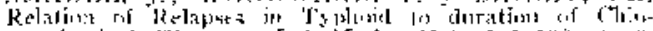

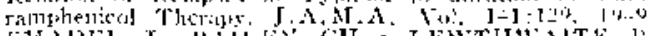

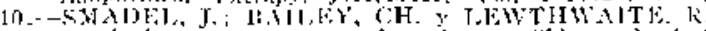

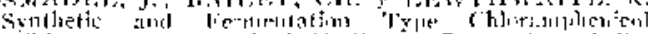

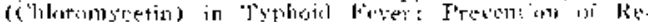

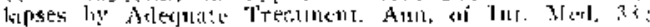
(]-1:), [950.

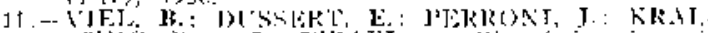

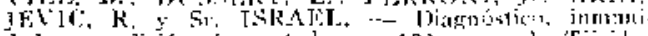

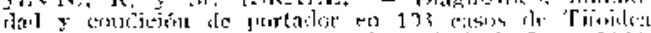

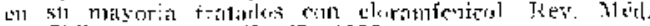
ile Cluile. Fol, su:458.267, 1952. 